Valero Obando, M. P., Vega Aponte, J. M., Almonacid Hurtado, P., y Támara Ayús, A. L. (2013). Determinantes de los ingresos laborales de los contadores públicos de la Universidad EAFIT. Revista Lebret (5). Bucaramanga, Colombia: Universidad Santo Tomás, pp. 357-375.

\title{
Determinantes de los ingresos laborales de los contadores públicos de la Universidad EAFIT, Colombia
}

\section{Determinants of Incomes of Public Accountants at EAFIT-Colombia}

\section{Resumen}

Paula María Almonacid Hurtado Armando Lenin Támara Ayús ${ }^{2}$ María Patricia Valero Obando ${ }^{3}$ Javier Mauricio Vega Aponte 4

El objetivo principal de este artículo es presentar los resultados de una investigación cuyo propósito fue establecer los determinantes de los salarios de los graduados de Contaduría Pública de la Universidad EAFIT, partiendo de la hipótesis que son el nivel de educación y la experiencia laboral acumulada, los principales factores que inciden en los salarios de los contadores. Se realiza un estudio de los principales indicadores del mercado laboral como son las tasas de ocupación, desempleo y subempleo y a través de una ecuación minceriana se muestra cuáles son los principales factores que inciden en una mejor remuneración salarial.

\section{Palabras clave:}

Contaduría Pública, Remuneración Salarial, Ecuación Minceriana

\section{Códigos de Clasificación JEL: J00, J001}

\begin{abstract}
The main purpose of this research is to identify the determinants of the wages of Certified Public Accountants (CAPs), who graduated from EAFIT University. As working hypothesis, we assume that the level of education and the years of professional experience are the main factors affecting wages. We estimate the Mincer equation, controlling for socioeconomic and employment characteristics of the alumni of the Accountancy program. The results obtained by the regression model show that the main factors affecting wages are the position, the fact of being employed by multinational companies, the fact of being male, the fact of being involved in an emotional relationship (currently or recently), and having a longer professional experience in terms of years.
\end{abstract}

\section{Keywords}

Certified Public Accountants (CAPs), Wages, Mincer Equation.

1 Economista EAFIT. Magíster en Ciencias Económicas de la Universidad de Montreal. Profesor-Investigador de la Universidad EAFIT Colombia. palmona1@eafit.edu.co

2 Economista Universidad de Antioquia. Especialista en Diseño y Evaluación de Proyectos Universidad del Norte. Magíster en Finanzas de la Universidad EAFIT. Profesor-Investigador de la Universidad EAFIT Colombia atamaraa@eafit.edu.co

3 Economista Universidad EAFIT. Correo electrónico: monitav_4@ hotmail.com

4 Economista, Especialista en Finanzas, Universidad EAFIT. Analista Riesgo de Crédito y Mercado, Serfinco S.A. Comisionistas de Bolsa. Correo electrónico: javiaponte@ hotmail.com 


\section{Introducción}

El creciente interés en temas de diferenciación salarial ha evidenciado la necesidad de analizar la importancia que conlleva el desarrollo del capital humano y el concepto de educación, visto como una inversión a largo plazo de la cual se espera recibir un rendimiento. La decisión de un individuo de aumentar su grado de escolaridad se traduciría en un aumento de su productividad, hecho que incrementaría a su vez sus ingresos laborales futuros.

En este sentido, el objetivo principal de la investigación que da origen al artículo hallar los determinantes de los salarios de los graduados de Contaduría Pública de la Universidad EAFIT, partiendo de la hipótesis que son el nivel de educación y la experiencia laboral acumulada, los principales factores que inciden en los salarios de los contadores, tal como se evidencia en el trabajo realizado por la Oficina de Planeación Integral acerca del impacto social del programa de pregrado de contaduría pública de la Universidad, y de acuerdo con los planteamientos mencionados por la Teoría del Capital Humano.

Los resultados arrojados por este estudio son relevantes en la medida en que permiten proporcionar una idea acerca de la dinámica del mercado de trabajo de grupos de la población específicos (en este caso individuos con un mismo título profesional), lo cual es de suma importancia, teniendo en cuenta que la remuneración salarial es uno de los aspectos de las condiciones de trabajo que más inciden en la vida de los trabajadores. Adicionalmente, los graduados tendrán una idea clara acerca de los rendimientos obtenidos por su educación.

Con el fin de llevar a cabo esta investigación, se realizó en primera instancia, un análisis de los principales indicadores del mercado laboral de los contadores públicos de la Universidad EAFIT, como son las tasas de ocupación, desempleo y subempleo; seguidamente se incluyó un análisis estadístico descriptivo de las variables más relacionadas con los cambios en los salarios y, finalmente, se estimó, mediante el método de Mínimos Cuadrados Ordinarios, una ecuación minceriana a la cual se le adicionaron otras variables socioeconómicas y laborales de los graduados, a fin de controlar por estos aspectos. Dichos procedimientos tuvieron como marco de referencia la Teoría del Capital Humano, las críticas que se le han hecho en cuanto a los posibles sesgos en los parámetros, y las formas propuestas por la literatura para corregirlas.

La información acerca de la población objeto de estudio (411 graduados del programa de Contaduría Pública de la Universidad EAFIT), se obtuvo a partir de una encuesta electrónica llevada a cabo por la Oficina de Planeación Integral de la Universidad desde el 7 de marzo hasta el 3 de mayo de 2011. La muestra fue recolectada de forma probabilística, con la restricción de ser estadísticamente representativa por estratos, según el año de graduación y el género. 
El presente trabajo se encuentra dividido en cinco secciones, siendo ésta introducción la primera de ellas seguida de una sección en la que se expone el marco teórico en el cual se referenció el trabajo. Posteriormente, se realiza la especificación y presentación de los modelos econométricos a estimar, con el fin de darle cabida a la cuarta sección, donde se materializa la descripción de los datos y el análisis de resultados en las regresiones econométricas. Por último, se exponen las conclusiones partiendo del análisis de los resultados encontrados.

\section{Marco teórico}

Existe una amplia literatura en el ámbito nacional e internacional que ha permitido analizar y cuantificar los determinantes de los ingresos de los trabajadores. En el caso específico de esta investigación, se tomó como referencia la teoría del capital humano para el análisis de los salarios de los contadores públicos de la Universidad EAFIT. De acuerdo con los planteamientos de dicha teoría, los trabajadores con condiciones socioeconómicas y culturales heterogéneas logran obtener un aumento en su productividad laboral, y por tanto, en sus ingresos laborales, mediante la acumulación de capital humano (Salas, 2003).

En este sentido, el concepto de capital humano es definido, bajo la luz de la teoría expuesta, como el conjunto de conocimientos y factores que generan habilidades y competencias a las personas. (Cardona, Montes, Vásquez, y Villegas, 2007). Entre los autores pioneros de la teoría del capital humano se encuentra Jacob Mincer que en 1974 realizó un modelo basado en la lógica económica de los individuos en el mercado laboral, mediante el cual analizó cómo la capacitación en el trabajo es causa de aumento en los salarios, y por tanto, de aumento en el nivel de ingresos de los individuos. En este sentido, los salarios se incrementan en la medida en que aumentan las cualificaciones de los individuos, es decir, en la medida en que el individuo se especializa en su labor (Cardona, Montes, Vásquez, \& Villegas, 2007). A continuación se presenta de forma un poco más detallada la lógica del planteamiento de Mincer (1974), así como la manera en que éste estima los retornos de factores como: educación, calidad en educación, e impacto de la experiencia de los trabajadores sobre el salario a partir de la llamada "ecuación minceriana".

\subsection{Función de Ganancias de Mincer}

A través de esta función de ganancias elaborada por Mincer (1974), es posible estimar el rendimiento de las inversiones educativas y profesionales, mediante la valoración del rendimiento del capital humano y el análisis de la estructura de ganancias de los individuos. En este sentido, las decisiones de inversión en educación se encuentran basadas en dos criterios fundamentales: el costo de oportunidad de la persona, y el horizonte de utilización de su stock de capital humano, que a su vez influencia el rendimiento de la inversión (Lassibille \& Navarro Gómez, 2004). 
De acuerdo con (Mincer, 1974) la vida laboral de cualquier individuo debe pasar por tres fases de un mismo ciclo. Un resumen del ciclo de vida laboral desarrollado por (Mincer, 1974) puede observarse en la siguiente Tabla.

Tabla 1. Resumen de la función de ganancias

\begin{tabular}{cccc}
\hline Fase del ciclo & $\begin{array}{c}\text { Proporción de renta } \\
\text { Invertida en formación }\end{array}$ & $\begin{array}{c}\text { Renta percibida en el } \\
\text { período t }\end{array}$ & $\begin{array}{c}\text { Rentabilidad de la } \\
\text { inversión en el período t }\end{array}$ \\
\hline Primera fase & La mayor proporción & Creciente & La mayor \\
\hline Segunda fase & Proporción intermedia & En desaceleración & En desaceleración \\
\hline Tercer fase & Nula & Estable o decrece & Estable o decreciente \\
\hline
\end{tabular}

Fuente: Autores.

En conclusión, siguiendo el razonamiento de Mincer (citado por Lassibille y Navarro Gómez, 2004) existe una relación directa entre los ingresos percibidos por una persona en determinado momento de su vida laboral y el capital acumulado hasta ese momento. El perfil de ganancias descrito en esta sección puede ser expresado matemáticamente mediante lo que se denomina función de rentas de la siguiente manera:

$$
Y_{t=} E_{t-} C_{t}
$$

Donde $Y_{t}$ es la renta neta, $E_{t}$ es la renta del periodo t observada antes de la decisión de inversión en capital humano y $C_{t}$ es el monto de las inversiones expresadas en términos monetarios. Asimismo, si se cuenta con una tasa de rendimiento de la inversión de capital humano $\left(\mathrm{r}_{\mathrm{t}-1}\right)$ de forma rezagada, es decir, para el periodo $(\mathrm{t}-1)$. De acuerdo con esto, la renta del año t se expresa de la siguiente forma:

$$
E_{t=} E_{t-1}+r_{t-1} C_{t-1}
$$

De forma recursiva se tiene:

$$
E_{t=} E_{0}+\sum^{t-1} r j C_{j}
$$

Donde $E_{0}$ representa la remuneración inicial del individuo, de esta forma la cantidad invertida en el periodo $t, C_{t}$, es una proporción $K_{t}$ de renta bruta $E_{i}$ :

$$
K_{t=} C_{t} / E_{t}
$$

La cual se puede expresar de forma logarítmica de la siguiente manera:

$$
\operatorname{In} E_{t}=\operatorname{In} E_{0}+\sum^{t-1} r j K j
$$


Esta ecuación muestra que la renta bruta en el periodo t, depende de la renta que corresponde a las capacidades innatas del individuo, y de las inversiones en educación escolar y profesional llevada a cabobo por un individuo (Lasibille y Navarro, 2004).

\subsection{Modelo Minceriano de Salarios}

El modelo minceriano de los salarios se centra en la función de ingresos obtenida por Mincer (1974) donde se pretende analizar la relación existente entre la inversión en capital humano y las diferencias salariales de los individuos. Por esta razón esta ecuación ha sido esencial a la hora de corroborar empíricamente los planteamientos de la teoría del capital humano.

En acorde con este modelo, estimado por el método de Mínimos Cuadrados Ordinarios (MCO), el log del ingreso individual (y) dado determinado tiempo puede ser descompuesto en una función de un término lineal de la educación y un término cuadrático de la experiencia (Card, 1999):

$$
\operatorname{In} Y=\beta_{0}+\beta_{1} S+\beta_{2} X+\beta_{3} X^{2}+u
$$

Donde In $Y$ es el logaritmo de las rentas salariales, $\mathrm{S}$ son los años de educación, $\mathrm{X}$ es la experiencia (años) y $\mathrm{X}^{2}$ los años de experiencia al cuadrado. El coeficiente $\beta_{1}$ de escolaridad, representa la estimación de la tasa de rendimiento en educación, la cual se supone como constante. Por otro lado, los coeficientes $\beta_{2}$ y $\beta_{3}$ referentes a la experiencia laboral (positivo y negativo, respectivamente), representan la concavidad de los ingresos (Card, 1999).

Con el fin de obtener la ecuación anterior, es necesario tener en cuenta tres de los supuestos propuestos por Mincer (1974) los cuales son descritos por Hidalgo (1999):

- Los costos de la educación son aquellos que dejan de percibirse durante la época de formación.

- Los individuos inician su vida laboral inmediatamente después de terminar sus estudios.

- La vida laboral de los individuos (n años), es independiente a sus años de estudios.

\section{Metodología}

El presente trabajo sigue un diseño explicativo - descriptivo. En primera medida, se constituye como explicativo debido a que se busca encontrar razones o causas por las cuales el salario de los Contadores Públicos varía en el mercado laboral, así 
como el porqué de la ocurrencia de dichos cambios y las condiciones en las cuales se presenta. Adicionalmente, el estudio se caracteriza como descriptivo, dado que los datos encontrados sirven para analizar cómo se manifiestan los determinantes de los salarios de los Contadores Públicos, detallándose mediante estadística descriptiva las características de los graduados como son sus condiciones laborales y socioeconómicas, las cuales permiten complementar el análisis de tipo explicativo. Adicionalmente, cabe anotar que la investigación es no experimental, ya que se realiza observando y analizando todas las características que la encuesta registra, sin tener que manipular variables de manera deliberada.

\subsection{Población estudiada}

El objeto de análisis de este estudio son los graduados del programa de Contaduría Pública de la Universidad EAFIT durante el período 1990-2010, los cuales son en total 411 individuos (Oficina de Planeación EAFIT). Su distribución por género y por edades, así como los tamaños de muestra representativos por estratos y la muestra actual se observan a continuación en las Tablas 2 y 3. Se tomó un nivel de confiabilidad del $95 \%$, un margen de error del $5 \%$, y los valores para p y para q de 0.92 y 0.08 respectivamente.

Tabla 2. Tamaños de muestra representativos por rangos de edades en años

\begin{tabular}{cccccccc}
\hline Rangos de edades & \multicolumn{2}{c}{ Población } & \multicolumn{2}{c}{$\begin{array}{c}\text { Muestra } \\
\text { representativa }\end{array}$} & Muestra actual & $\begin{array}{c}\text { Dif. M. actual } \\
\text { y M. rep. }\end{array}$ \\
\hline Entre 23 y 27 & 63 & $15,33 \%$ & 14 & $3,31 \%$ & 37 & $9,00 \%$ & 23 \\
\hline Entre 28 y 32 & 102 & $24,82 \%$ & 22 & $5,36 \%$ & 34 & $8,27 \%$ & 12 \\
\hline Entre 33 y 37 & 138 & $33,58 \%$ & 30 & $7,25 \%$ & 34 & $8,27 \%$ & 4 \\
\hline Entre 38 y 42 & 58 & $14,11 \%$ & 13 & $3,05 \%$ & 22 & $5,35 \%$ & 9 \\
\hline Entre 43 y 47 & 28 & $6,81 \%$ & 6 & $1,47 \%$ & 11 & $2,68 \%$ & 5 \\
\hline Entre 48 y 52 & 14 & $3,41 \%$ & 3 & $0,74 \%$ & 11 & $2,68 \%$ & 8 \\
\hline Entre 53 y 57 & 5 & $1,22 \%$ & 1 & $0,26 \%$ & 5 & $1,22 \%$ & 4 \\
\hline Entre 58 y 62 & 1 & $0,24 \%$ & 0 & $0,05 \%$ & 0 & $0,00 \%$ & 0 \\
\hline Entre 63 y 67 & 1 & $0,24 \%$ & 0 & $0,05 \%$ & 0 & $0,00 \%$ & 0 \\
\hline Entre 68 y 72 & 1 & $0,24 \%$ & 0 & $0,05 \%$ & 0 & $0,00 \%$ & 0 \\
\hline Total & $\mathbf{4 1 1}$ & $\mathbf{1 0 0 , 0 0 \%}$ & $\mathbf{8 8 , 6 9}$ & $\mathbf{2 1 , 5 8 \%}$ & $\mathbf{1 5 4}$ & $\mathbf{3 7 , 4 7 \%}$ & $\mathbf{6 5}$ \\
\hline
\end{tabular}

Fuente: Oficina de Planeación. Encuesta de Seguimiento a Egresados, Universidad EAFIT, 2011. Tabla 3. Tamaños de muestra representativos por género 


\begin{tabular}{|c|c|c|c|c|c|c|c|}
\hline Género & \multicolumn{2}{|c|}{ Población } & \multicolumn{2}{c|}{$\begin{array}{c}\text { Muestra } \\
\text { representativa }\end{array}$} & \multicolumn{2}{c|}{ Muestra actual } & $\begin{array}{c}\text { Dif. M. actual } \\
\text { y M. rep. }\end{array}$ \\
\hline Femenino & 287 & 62,3 & 62 & $15,07 \%$ & 96 & $23,36 \%$ & 34 \\
\hline Masculino & 124 & 37,7 & 27 & $6,51 \%$ & 58 & $14,11 \%$ & 31 \\
\hline Total & 411 & 100,0 & 88,69 & $21,58 \%$ & 154 & $37,47 \%$ & 65 \\
\hline
\end{tabular}

Fuente: Oficina de Planeación. Encuesta de Seguimiento a Egresados, Universidad EAFIT, 2011.

\subsection{Instrumento de recolección de la información}

La información se obtuvo a partir de una fuente primaria, es decir que se aplicó - mediante una aplicación web desarrollada para el uso de la Universidad llamada SEVEN - un instrumento (formulario de encuesta) diseñado principalmente por el Ministerio Nacional de Educación para el seguimiento de los graduados de las Universidades colombianas, el cual ha sido utilizado en varias ocasiones por la Oficina de la Dirección de Planeación de la Universidad EAFIT.

Dicho cuestionario comprende las siguientes secciones: datos generales del graduado, situación laboral, trayectoria académica y profesional del graduado, evaluación de las competencias, evaluación del programa y de la institución y conocimiento acerca de EAFIT. Dado que la encuesta en línea es "inteligente", el número de preguntas de cada sección puede variar para cada graduado dependiendo de su situación en relación con el mercado laboral, es decir, si es ocupado (y dentro de esta subsección, si es empleado, trabajador por cuenta propia o empresario), desocupado o inactivo.

\subsection{Análisis de información y especificación de modelos de regresión}

El análisis de la información se realizó en dos etapas: en la primera de estas se llevó a cabo una caracterización socioeconómica y laboral de los individuos que componen la muestra, mediante herramientas de estadística descriptiva y el análisis de indicadores laborales. Adicionalmente, se relacionó el salario promedio devengado con cada una de las características laborales y socioeconómicas de los contadores encuestados, con el fin de lograr una primera aproximación a los factores determinantes de los salarios. La segunda etapa del análisis fue realizada a partir de un modelo de regresión que sufrió cambios, en lo que tiene que ver con su especificación, a lo largo de las estimaciones para llegar a una estimación agregada que permitiera cumplir el principal objetivo de la investigación. A continuación se profundiza en el procedimiento llevado a cabo en esta etapa de análisis. 


\subsubsection{Modelos de Regresión.}

En un primer momento, se estimó la ecuación original minceriana donde el logaritmo del ingreso es la variable dependiente (en el caso específico de esta investigación se tomó como variable dependiente el logaritmo natural del salario mensual de los individuos bajo estudio), y las variables independientes son los años de educación, la experiencia laboral y el cuadrado de esta.

$$
\log \left(\text { Ing }_{i}\right)=\beta_{0}+\beta_{1} \text { Educ }+\beta_{2} \text { Experiencia }+\beta_{3} \text { Experiencia }^{2}+\mu
$$

Donde el coeficiente asociado a la variable educación es la tasa de rendimiento de un año adicional de educación. Posteriormente se tiene la experiencia, en el caso de esta investigación fue medida a partir del número de años de experiencia profesional y, por último, se incluyó el término experiencia al cuadrado, que indica la concavidad de la función de la experiencia.

Como segunda medida, con el fin de identificar los principales factores que determinan los salarios de los contadores de la Universidad EAFIT, desde la teoría del capital humano y con una especificación más robusta, se estimó por medio del método de Mínimos Cuadrados Ordinarios una ecuación tipo Mincer (1974), incluyendo variables de tipo socioeconómico como variables de control. Específicamente el modelo que se desarrolló fue el siguiente:

$$
\begin{gathered}
\log \left(\text { Ing }_{i}\right)=\beta_{0}+\beta_{1} \text { Edad }+\beta_{2} \text { Edad }^{2}+\beta_{3} \text { Genero }+\beta_{4} \text { Estcivil }+\beta_{2} \text { Educ }+ \\
\beta_{6} \text { Experiencia }+\beta_{7} \text { Experiencia }^{2}+\beta_{8} \text { Estrato }+\mu
\end{gathered}
$$

Finalmente, teniendo en cuenta las críticas realizadas a la ecuación planteada por Mincer (1974) en cuanto a que en ésta no se tienen en cuenta aspectos del lado de la demanda de trabajo, se incluyeron entonces algunas variables de este tipo entre las cuales se encuentran: el tipo de empleador, sea empresa pública o privada, ámbito de la empresa, multinacional, nacional, regional o local, y los cargos dentro de las organizaciones. En el caso específico de esta investigación, resultaron significativas las siguientes: D_Multinacional, D_Gerente y D_Director.

$$
\begin{gathered}
\log \left(\text { Ing }_{i}\right)=\beta_{0}+\beta_{1} \text { Experiencia }+\beta_{2} \text { Experiencia }^{2}+\beta_{3} \text { Educ }+\beta_{4} \text { Edad }+ \\
\beta_{5} \text { Edad }^{2}+\beta_{6} \text { Estcivil }+\beta_{7} \text { Genero }+\beta_{8} \text { Estrato }+\beta_{9} \text { Multinacional }+ \\
\beta_{10} D_{-} \text {Director }+\mu
\end{gathered}
$$

El planteamiento de este modelo permitió conocer las variables que son significativas en términos estadísticos para explicar los salarios de los graduados del programa de pregrado de Contaduría Pública de la Universidad EAFIT, así como el incremento porcentual en el salario de los individuos, por cada año de educación 
formal y experiencia laboral adicionales, lo mismo que el efecto de algunas de las variables socioeconómicas y laborales que caracterizan la población bajo estudio.

\subsubsection{Sesgo en los resultados esperados y formas de corregirlo.}

En la aplicación empírica de este modelo se han evidenciado una serie de errores de sesgo en la estimación de parámetros, razón por la cual no ha dejado de ser criticado. Como señala Hidalgo (1999), los problemas asociados a la estimación del modelo minceriano pueden diferenciarse en tres categorías: primero, problemas de fuentes de datos (omisión de la variable habilidad en la especificación de la función); segundo, de endogeneidad, y por último, dificultades en la medición de variables (Salas, 2001).

En lo que concierne a la última fuente de sesgo mencionada en el párrafo anterior, se tiene que, tanto la medición de la educación como de la experiencia, generalmente están sujetas a error. Según Tenjo, Ribero y Bernat (2005)

En la mayoría de las bases de datos disponibles no hay buenas medidas de experiencia relevante, en muchas ocasiones la única medida que se puede obtener es una medida de experiencia potencial (Edad menos años de educación menos 5). El tener una medida que contiene errores de medición hace que el supuesto de independencia del modelo de regresión se viole y que los estimativos de los coeficientes del modelo sean sesgados ( $\mathrm{p} 10$ ).

Finalmente, se encuentra el conocido sesgo de selección muestral que, según Tenjo et. al ( 2005) consiste en el sesgo que se produce en los estimativos de los coeficientes por no incluir el salario de aquellas personas que no laboran en el momento de la medición.

En cuanto a la primera posibilidad de sesgo descrita en el párrafo anterior, el procedimiento que se siguió fue la inclusión de la variable "promedio crédito acumulado de la carrera" como variable proxy para la habilidad del individuo. Seguidamente, en lo que se refiere a la forma de medición de las variables "educación" y "experiencia", se tomaron las medidas: "número de años de educación formal" y "número de meses de experiencia laboral", las cuales pueden considerarse como medidas confiables y relevantes de los conceptos que se desean medir. Finalmente, en lo que se refiere al sesgo de selección muestral, se realizó el procedimiento planteado por Prada y Vélez (2006) basado en Heckman (1979), mediante el cual se plantea una estimación en dos etapas: en la primera se estima un modelo probit que calcula la probabilidad de los individuos pertenecientes a la población en edad de trabajar (PET) de hacer parte del mercado laboral usando la siguiente ecuación de participación laboral:

$$
\begin{aligned}
& P_{i}=\beta_{0}+\beta_{1} \text { Experiencia }+\beta_{2} \text { Experiencia }{ }^{2}+\beta_{3} \text { Educ }+\beta_{4} \text { Edad }+ \\
& \beta_{5} \text { Edad }^{2}+\beta_{6} \text { Estcivil }+\beta_{7} \text { Genero }+\beta_{8} \text { Estrato }+\beta_{9} \text { Hijos }
\end{aligned}
$$


Luego de haber obtenido este resultado se estima el "inverso de la razón de Mills"

$$
\lambda=\left[\varnothing\left(Z_{i}\right)\right]:\left[1-\varphi\left(-Z_{i}\right)\right]
$$

Donde: $Z_{i}$ proviene de la ecuación del probit; los parámetros $\varnothing$ y $\varphi$ son la función de densidad de una variable aleatoria normal estándar y la función de distribución acumulativa, respectivamente. $\lambda$ se incluye como regresor en la función original del modelo global durante la segunda etapa de estimación para corregir el sesgo. Posteriormente, en la segunda etapa de estimación, se incluye el valor de $\lambda$ en la ecuación de ingresos, como una más de las variables explicativas, para luego ser estimada mediante el método de Mínimos Cuadrados Ordinarios.

\section{Aplicación empírica y análisis de resultados}

Con el fin de responder a la hipótesis planteada respecto que la educación formal y la experiencia laboral son los principales factores que inciden en los salarios percibidos por los contadores públicos de la Universidad EAFIT, se procedió al análisis estadístico de los datos socioeconómicos y laborales.

\subsection{Características socioeconómicas de los graduados}

A continuación se muestran las principales características socioeconómicas que describen la muestra de contadores públicos eafitenses encuestados.

Tabla 4. Características socioeconómicas

\begin{tabular}{|c|c|c|c|}
\hline Variable & Categorías & Frecuencia & Porcentaje \\
\hline \multirow{3}{*}{ Género } & Femenino & 96 & $62,34 \%$ \\
\hline & Masculino & 58 & $37,66 \%$ \\
\hline & Total & 154 & $100,00 \%$ \\
\hline \multirow{6}{*}{ Estado civil actual } & Soltero(a) & 73 & $47,40 \%$ \\
\hline & Casado(a) & 65 & $42,21 \%$ \\
\hline & Separado(a)/Divorciado(a) & 11 & $7,14 \%$ \\
\hline & Unión libre & 4 & $2,60 \%$ \\
\hline & Viudo(a) & 1 & $0,65 \%$ \\
\hline & Total & 154 & $100,00 \%$ \\
\hline \multirow{6}{*}{ Número de hijos } & 0 & 101 & $65,58 \%$ \\
\hline & 1 & 25 & $16,23 \%$ \\
\hline & 2 & 24 & $15,58 \%$ \\
\hline & 3 & 3 & $1,95 \%$ \\
\hline & 4 & 1 & $0,65 \%$ \\
\hline & Total & 154 & $100,00 \%$ \\
\hline \multirow{6}{*}{ Estrato de la vivienda } & 5 & 50 & $32,47 \%$ \\
\hline & 4 & 45 & $29,22 \%$ \\
\hline & 6 & 30 & $19,48 \%$ \\
\hline & 3 & 25 & $16,23 \%$ \\
\hline & 2 & 4 & $2,60 \%$ \\
\hline & Total & 154 & $100,00 \%$ \\
\hline
\end{tabular}

Fuente: Oficina de Planeación. Encuesta de Seguimiento a Egresados, Universidad EAFIT, 2011. 
A partir de la Tabla anterior se observa que la población estudiada se compone de mujeres en su gran mayoría. Adicionalmente, en cuanto al estado civil la muestra se divide casi proporcionalmente entre quienes se encuentran solteros y quienes están casados. No obstante, la gran mayoría no tiene hijos y viven en estratos altos, en orden de importancia, 5,4 y 6.

Tabla 5. Características socioeconómicas

\begin{tabular}{|c|c|c|c|}
\hline Variable & Categorías & Frecuencia & Porcentaje \\
\hline \multirow{8}{*}{$\begin{array}{l}\text { Edad (en número de } \\
\text { años) }\end{array}$} & Entre 23 y 27 & 37 & $24,03 \%$ \\
\hline & Entre 28 y 32 & 34 & $22,08 \%$ \\
\hline & Entre 33 y 37 & 34 & $22,08 \%$ \\
\hline & Entre 38 y 42 & 22 & $14,29 \%$ \\
\hline & Entre 43 y 47 & 11 & $7,14 \%$ \\
\hline & Entre 48 y 52 & 11 & $7,14 \%$ \\
\hline & Entre 53 y 57 & 5 & $3,25 \%$ \\
\hline & Total & 154 & $100,00 \%$ \\
\hline \multirow{7}{*}{$\begin{array}{l}\text { Grado de educación } \\
\text { más alto }\end{array}$} & Especialización & 62 & $40,26 \%$ \\
\hline & Diplomados & 38 & $24,68 \%$ \\
\hline & Maestría & 23 & $14,94 \%$ \\
\hline & Seminarios/Cursos & 19 & $12,34 \%$ \\
\hline & Ninguna & 10 & $6,49 \%$ \\
\hline & Otro Pregrado & 2 & $1,30 \%$ \\
\hline & Total & 154 & $100,00 \%$ \\
\hline \multirow{13}{*}{ Ciudad de residencia } & Medellín y su área metropolitana & 140 & $90,91 \%$ \\
\hline & Bogotá & 4 & $2,60 \%$ \\
\hline & Montería & 1 & $0,65 \%$ \\
\hline & Sincelejo & 1 & $0,65 \%$ \\
\hline & Rionegro & 1 & $0,65 \%$ \\
\hline & Miami & 1 & $0,65 \%$ \\
\hline & Riohacha & 1 & $0,65 \%$ \\
\hline & Quibdó & 1 & $0,65 \%$ \\
\hline & Andes & 1 & $0,65 \%$ \\
\hline & Barranquilla & 1 & $0,65 \%$ \\
\hline & Guarne & 1 & $0,65 \%$ \\
\hline & Valledupar & 1 & $0,65 \%$ \\
\hline & Total & 154 & $100,00 \%$ \\
\hline
\end{tabular}

Fuente: Oficina de Planeación. Encuesta de Seguimiento a Egresados, Universidad EAFIT, 2011.

Finalmente, se observa que la mayoría de los graduados estudiados se encuentran en un rango de edad entre 23 y 42 años, han realizado mayoritariamente cursos de especialización y maestría, y residen actualmente en la ciudad de Medellín y su área metropolitana. 


\subsubsection{Caracterización de las empresas y cargos.}

A continuación se describen los principales rasgos que caracterizan las empresas y los cargos de los graduados ocupados. En primer lugar, con respecto a la actividad económica de las organizaciones donde los contadores públicos eafitenses se encuentran laborando, se puede afirmar que gran parte de éstos están ubicados en empresas que se dedican a actividades financieras $(29,2 \%)$, el comercio $(18,3 \%)$ y la educación (16,2\%). Se destaca el hecho de que el grupo bajo estudio ha logrado desempeñarse laboralmente en 12 sectores de la economía como son el de agricultura, ganadería, caza y silvicultura $(1,4 \%)$; hoteles, restaurantes, bares y similares $(1,4 \%)$; explotación de minas y canteras $(1,4 \%)$.

En cuanto al tamaño de empresa, se puede ver que la gran mayoría de graduados encuestados $(65,6 \%)$ trabajan en empresas grandes, es decir, con más de 200 empleados; en segundo lugar, se ubican en empresas pequeñas $(17,6 \%)$, es decir, con un número de empleados entre uno y cincuenta y, finalmente, el restante número de graduados encuestados se encuentra ubicado en empresas medianas (16,8\%), o en otras palabras, en empresas que cuentan entre 51 y 200 empleados.

Por su parte, en lo que concierne a la organización, se destaca el ámbito multinacional el cual agrupa el 38,7\% de los graduados bajo estudio, seguido por el nacional (37\%), y a el municipal (12,7\%). En las organizaciones del ámbito regional sólo se ubican un $11,7 \%$ de los contadores públicos eafitenses que contestaron la encuesta.

Ahora bien, la información recogida acerca de los cargos y ocupaciones de los graduados en dichos cargos muestra que el $28,2 \%$ de los contadores públicos eafitenses son analistas, seguidos por el 15,5\% quienes son gerentes, el 10,6\% se encuentran en otros cargos no identificados dentro de las opciones de cargo presentadas en la encuesta, y el 9,9\% son directores. Estos resultados dan cuenta de la experticia y éxito de los contadores en sus respectivos puestos de trabajo. Por su parte, las ocupaciones que predominan entre los contadores públicos eafitenses son aquellas relacionados con contabilidad (27,5\%), finanzas (17,6\%), y auditoría $(14,8 \%)$.

\subsubsection{Análisis de los salarios.}

Por otra parte, al reconocer las actividades económicas mejor remuneradas en el mercado laboral de los contadores públicos, se logra observar que en los sectores privados y mixtos y en las industrias con un enfoque en servicios y explotación de minas los contadores obtienen mejores remuneraciones salariales (Tabla 5). 
Tabla 5. Salario por actividad económica y tipo de empresa

\begin{tabular}{lcccc}
\hline \multirow{2}{*}{$\begin{array}{c}\text { Actividad económica } \\
\text { de la organización }\end{array}$} & \multicolumn{3}{c}{ Tipo de empresa } \\
\cline { 2 - 5 } & Mixto & N/A & Privado & Público \\
\cline { 2 - 5 } & Promedio & Promedio & Promedio & Promedio \\
\hline Agricultura, Ganadería, Caza y Silvicultura & & 2.000 .000 & 2.600 .000 \\
\hline Comercio & & 2.980 .000 & 4.230 .625 \\
\hline Construcción & 3.650 .000 & 3.450 .000 & 4.000 .000 \\
\hline Educación & & & 11.750 .000 & \\
\hline Explotación de Minas y Canteras & 4.700 .000 & 3.642 .688 & 3.250 .000 \\
\hline Financieras & & & 3.596 .093 & 3.000 .000 \\
\hline Industrias Manufactureras & 5.000 .000 & 5.599 .334 \\
\hline Servicios & 5.950 .000 & 7.000 .000 & 3.870 .610 & 5.200 .000 \\
\hline
\end{tabular}

Fuente: Oficina de Planeación. Encuesta de Seguimiento a Egresados, Universidad EAFIT, 2011.

\subsection{Estimación del modelo}

Luego de una primera aproximación a los factores determinantes de los salarios de los contadores públicos eafitenses mediante el análisis descriptivo, se pasará ahora a establecer dichos determinantes mediante un análisis de tipo estadístico inferencial, específicamente mediante la estimación de tres modelos de regresión cuyas especificaciones tienen como base la ecuación de salarios propuesta por Mincer (1976).

En este sentido, se estimó mediante el método de Mínimos Cuadrados Ordinarios la ecuación de Mincer, posteriormente se plantea una ecuación que comprende como variables explicativas en adición a aquellas propuestas por Mincer, variables de control que recogen características de tipo socioeconómico de los individuos. Por último, se estima una ecuación de regresión que tuvo como base las variables de la ecuación minceriana pero que, además, incluyó variables de tipo socioeconómico y variables relacionadas con las características de demanda laboral de los individuos, como se verá con más detalle en el desarrollo de esta sección. Estos dos últimos modelos de regresión fueron igualmente estimados mediante el método de Mínimos Cuadrados Ordinarios.

Antes de presentar los resultados de la mencionada rutina de ecuaciones, se considera importante resaltar que, en primer lugar, se verificó mediante el método de estimación de Heckman en dos etapas, que el modelo a estimar no presentaba el problema de sesgo de selección muestral, el cual surge principalmente de la ausencia de aleatoriedad muestral, o selección muestral no aleatoria. En la primera etapa se estimó mediante el método de máxima verosimilitud, un modelo Probit con el fin de determinar la probabilidad de estar en la muestra, o en otras palabras, se estimó la ecuación de selección. Luego, a partir de la estimación de dicho Probit, se calculó el valor de lambda estimado, con lo cual se obtuvo, la probabilidad de estar en la muestra sobre la probabilidad de no encontrarse en ella. 
Finalmente, en la segunda etapa se estimó un modelo lineal con la variable dependiente continua (logaritmo natural del salario mensual) incluyendo lambda por el método MCO. Generalmente, el contraste del sesgo selección se realiza con una prueba de hipótesis de significancia individual de la inversa del Ratio de Mills. Si se rechaza la hipótesis nula, es decir, si el coeficiente que acompaña a la inversa del Ratio de Mills resulta ser significativo, existe un sesgo de selección y este puede ser corregido mediante este método de estimación; si por el contrario, el coeficiente no resulta significativo, lo ideal es estimar los coeficientes obtenidos mediante MCO.

En el caso específico de este estudio los resultados obtenidos de la ecuación de selección o, en otras palabras, de la primera etapa de la estimación, pueden observarse en la Tabla 6. Se considera importante resaltar que las variables independientes que hacen parte de este modelo son los años de educación, los años de experiencia profesional, los años de experiencia profesional al cuadrado, el género, la edad, la edad al cuadrado, el estado civil, y el número de hijos. De acuerdo con los resultados obtenidos, sólo la variable experiencia profesional resultó estadísticamente significativa para explicar la participación laboral de los contadores públicos eafitenses. De otro lado, se encontró que el término lambda (también llamado inverso de la razón de Mills) tampoco es estadísticamente significativo, y por tanto, se puede concluir que no existe un sesgo de selección, y que, en consecuencia, es posible estimar la rutina de ecuaciones planteada mediante el método MCO.

Tabla 6. Estimación primera etapa

\begin{tabular}{|c|c|c|c|c|c|c|c|}
\hline \multicolumn{4}{|c|}{$\begin{array}{l}\text { Heckman selection model }-- \text { two-step estimates } \\
\text { (regression model with sample selection) }\end{array}$} & \multirow{2}{*}{\multicolumn{2}{|c|}{$\begin{array}{l}\text { Number of obs } \\
\text { Censored obs } \\
\text { Uncensored obs } \\
\text { Wald chi2(3) } \\
\text { Prob > chi2 }\end{array}$}} & \multirow{2}{*}{$\begin{array}{l}= \\
= \\
= \\
= \\
=\end{array}$} & \multirow{2}{*}{$\begin{array}{r}152 \\
12 \\
140 \\
16.72 \\
0.0008\end{array}$} \\
\hline & & & & & & & \\
\hline & coef. & std. Err. & $\mathbf{z}$ & $P>|z|$ & \multicolumn{2}{|c|}{$[95 \%$ conf. } & Interva1] \\
\hline $\begin{array}{r}\text { Insalario } \\
\text { exper } \\
\text { educ } \\
\text { exper } 2 \\
\text {-cons }\end{array}$ & $\begin{array}{l}.0482662 \\
.0927651 \\
8.25 \mathrm{e}-06 \\
13.11449\end{array}$ & $\begin{array}{r}.0494002 \\
.0671099 \\
.0019207 \\
1.183683\end{array}$ & $\begin{array}{r}0.98 \\
1.38 \\
0.00 \\
11.08\end{array}$ & $\begin{array}{l}0.329 \\
0.167 \\
0.997 \\
0.000\end{array}$ & $\begin{array}{r}-.0485 \\
-.0387 \\
-.0037 \\
10.79\end{array}$ & $\begin{array}{l}5563 \\
7678 \\
7562 \\
9452\end{array}$ & $\begin{array}{r}.1450888 \\
.224298 \\
.0037727 \\
15.43447\end{array}$ \\
\hline $\begin{array}{r}\text { hijos } \\
\text { civi1 } \\
\text { genero } \\
\text { estrat } \\
\text { Edad } \\
\text { exper } \\
\text { educ } \\
\text { exper2 } \\
\text { edad2 } \\
\text { _cons }\end{array}$ & $\begin{array}{r}.1787374 \\
-.0732354 \\
.2121802 \\
-.4919103 \\
-.345443 \\
.3418286 \\
-.0402109 \\
-.0089818 \\
.0031963 \\
8.310131\end{array}$ & $\begin{array}{r}.3022155 \\
.4396223 \\
.3673809 \\
.5257348 \\
.2478294 \\
.137053 \\
.1088012 \\
.0043937 \\
.0030385 \\
4.785267\end{array}$ & $\begin{array}{r}0.59 \\
-0.17 \\
0.58 \\
-0.94 \\
-1.39 \\
2.49 \\
-0.37 \\
-2.04 \\
1.05 \\
1.74\end{array}$ & $\begin{array}{l}0.554 \\
0.868 \\
0.564 \\
0.349 \\
0.163 \\
0.013 \\
0.712 \\
0.041 \\
0.293 \\
0.082\end{array}$ & $\begin{array}{r}-.4135 \\
-.9348 \\
-.507 \\
-1.522 \\
-.8311 \\
.0732 \\
-.2534 \\
-.0175 \\
-.0027 \\
-1.06\end{array}$ & $\begin{array}{l}5941 \\
8793 \\
7873 \\
2332 \\
1798 \\
2096 \\
4573 \\
5932 \\
7591 \\
6882\end{array}$ & $\begin{array}{r}.7710689 \\
.7884085 \\
.9322335 \\
.538511 \\
.1402938 \\
.6104476 \\
.1730354 \\
-.0003703 \\
.0091517 \\
17.68908\end{array}$ \\
\hline mi11s 1 ambda & -.9874067 & .8168395 & -1.21 & 0.227 & -2.588 & 8383 & .6135693 \\
\hline $\begin{array}{r}\text { rho } \\
\text { sigma } \\
\text { 1ambda }\end{array}$ & $\begin{array}{r}-1.00000 \\
.98740666 \\
-.98740666\end{array}$ & .8168395 & & & & & \\
\hline
\end{tabular}

Fuente: Autores. Salida STATA. 
Siguiendo con la metodología propuesta, se procedió a la estimación de la ecuación de Mincer para la muestra estudiada (Tabla 7). Los resultados de dicha estimación mostraron que todas las variables, a excepción de la experiencia profesional al cuadrado, resultaron significativas para explicar el logaritmo natural de los salarios mensuales de los contadores bajo estudio. Ahora bien, dado que la variable experiencia profesional al cuadrado no resultó significativa, es decir que la función de la experiencia no es parabólica, o lo que es lo mismo, que el rendimiento de los salarios comienza a decrecer a partir de cierto número de años de experiencia, se procedió a excluirla del modelo.

Tabla 7. Estimación de la Ecuación de Mincer

\begin{tabular}{|c|c|c|c|c|c|c|}
\hline Linear regres & & & & & $\begin{array}{l}\text { Number of obs } \\
\text { F( 2, 137) } \\
\text { Prob > F } \\
\text { R-squared } \\
\text { Root MSE }\end{array}$ & $\begin{array}{rr}= & 140 \\
= & 29.35 \\
= & 0.0000 \\
= & 0.3410 \\
= & .49494\end{array}$ \\
\hline Insalario & coef. & $\begin{array}{l}\text { Robust } \\
\text { Std. Err. }\end{array}$ & $t$ & $P>|t|$ & [95\% conf. & Interval] \\
\hline $\begin{array}{l}\text { exper } \\
\text { educ } \\
\text { cons }\end{array}$ & $\begin{array}{l}.0527753 \\
.0773996 \\
13.20034\end{array}$ & $\begin{array}{l}.0072687 \\
.0346929 \\
.6019688\end{array}$ & $\begin{array}{r}7.26 \\
2.23 \\
21.93\end{array}$ & $\begin{array}{l}0.000 \\
0.027 \\
0.000\end{array}$ & $\begin{array}{l}.0384019 \\
.0087968 \\
12.00999\end{array}$ & $\begin{array}{r}.0671487 \\
.1460025 \\
14.39069\end{array}$ \\
\hline
\end{tabular}

Fuente: Autores. Salida STATA.

En cuanto al peso de los coeficientes, se puede afirmar que la variable educación es la que más se destaca. En el momento no se describirá el cambio porcentual de la variable dependiente en relación con el cambio en una unidad en la variable independiente (semi-elasticidad), ya que esto se dejará para los resultados de la regresión final. Por ahora, sólo se tiene como objetivo mostrar la sensibilidad de los coeficientes del modelo en la medida en que se incluyen distintas variables de control.

A continuación, se realiza la estimación de la ecuación de Mincer incluyendo variables de tipo personal como variables de control (Tabla 8). A partir de los resultados arrojados, es posible afirmar que todas las variables incluidas son estadísticamente significativas para explicar los salarios de los contadores públicos eafitenses a un $5 \%$ de significancia. Entre las variables que presentan una mayor influencia en el comportamiento de los salarios, hasta ahora, se encuentran, en orden de importancia: el estrato, el estado civil y el género ${ }^{5}$. Por su parte, las variables experiencia y años de educación parecen no ser tan influyentes a la hora de determinar los salarios de la población estudiada (presentando un mayor peso la educación que la experiencia).

5 Se intentó incluir en el grupo de variables explicativas las variables promedio acumulado (como variable proxy para la habilidad del graduado), número de hijos y edad, sin embargo estas no resultaron significativas. 
Ahora bien, en lo que tiene que ver con la sensibilidad o cambio de los coeficientes luego de la inclusión de las variables socioeconómicas como controles, se encontró que ambos coeficientes disminuyeron considerablemente.

Tabla 8. Estimación de la ecuación de Mincer incluyendo variables de tipo personal

\begin{tabular}{|c|c|c|c|c|c|c|}
\hline \multicolumn{5}{|c|}{ Linear regression } & $\begin{array}{l}\text { Number of obs } \\
\text { FC 5, 134) } \\
\text { Prob }>\text { F } \\
\text { R-squared } \\
\text { Root MSE }\end{array}$ & \multirow{2}{*}{$\begin{array}{lr}= & 140 \\
= & 17.23 \\
= & 0.0000 \\
= & 0.4439 \\
= & .45971\end{array}$} \\
\hline Tnsalario & coef. & $\begin{array}{l}\text { Robust } \\
\text { Std. Err. }\end{array}$ & $\mathrm{t}$ & $P>|t|$ & [95\% conf. & \\
\hline $\begin{array}{r}\text { exper } \\
\text { educ } \\
\text { civi1 } \\
\text { genero } \\
\text { estrat } \\
\text { _cons }\end{array}$ & $\begin{array}{r}.0427411 \\
.0666689 \\
-.2150558 \\
.2127412 \\
.3171858 \\
13.25313\end{array}$ & $\begin{array}{l}.0079051 \\
.0319455 \\
.0909771 \\
.0805148 \\
.1070108 \\
.5638563\end{array}$ & $\begin{array}{r}5.41 \\
2.09 \\
-2.36 \\
2.64 \\
2.96 \\
23.50\end{array}$ & $\begin{array}{l}0.000 \\
0.039 \\
0.020 \\
0.009 \\
0.004 \\
0.000\end{array}$ & $\begin{array}{r}.0271062 \\
.0034863 \\
-.3949927 \\
.0534969 \\
.1055371 \\
12.13792\end{array}$ & $\begin{array}{r}.058376 \\
.1298515 \\
-.0351189 \\
.3719855 \\
.5288346 \\
14.36834\end{array}$ \\
\hline
\end{tabular}

Fuente: Autores. Salida STATA.

Finalmente, se analizan los resultados de la estimación del modelo más completo, el cual incluye variables de tipo socioeconómico y de demanda laboral (Tabla 9). Según estos, la variable años de educación no resultó estadísticamente significativa, lo cual quiere decir que la educación recibida por los sujetos bajo estudio no influye en el comportamiento de los salarios de los mismos, y por esta razón, dicha variable fue retirada del modelo.

Ahora bien, de acuerdo con los resultados los factores que más pesan en el comportamiento de los salarios son en orden de importancia: quienes ocupan un cargo de director (de acuerdo con el valor del coeficiente de esta variable, los individuos que ocupan cargos de directores ganan un 70,59\% más que el resto), quienes ocupan un cargo de gerente (los cuales perciben una prima de 62,84\%), quienes trabajan para empresas multinacionales (los cuales reciben una prima en sus salarios de 33,31\%), quienes viven en estratos altos (los cuales perciben una prima de 22,84\%), los hombres (que perciben un 19,92\% más de salario que las mujeres), quienes no son solteros, o en otras palabras, quienes se encuentran o se encontraban en una relación sentimental (que perciben una prima en sus salarios de 17,77\%), y finalmente, se puede afirmar que cada año adicional de experiencia proporciona una retribución en los salarios de 4,07\%.

6 El valor de este coeficiente no coincide con el del cuadro ya que la forma funcional de la variable dependiente (logaritmo natural) no permite que las semi-elasticidades puedan ser interpretadas directamente en todos los casos. Esto debido a que en la medida en que el $\log (\mathrm{y})$ se hace grande, la aproximación porcentual se hace cada vez más imprecisa. Con el fin de subsanar esta desventaja, es posible utilizar la fórmula $100 \mathrm{x}(E X P(\widehat{\beta})-1)$ para realizar una interpretación correcta. 
Tabla 9. Estimación del modelo completo

\begin{tabular}{|c|c|c|c|c|c|c|}
\hline Linear regres & & & & & $\begin{array}{l}\text { Number of obs } \\
\text { F( 7, 132) } \\
\text { Prob > F } \\
\text { R-squared } \\
\text { Root MSE }\end{array}$ & $\begin{array}{rr}= & 140 \\
= & 38.98 \\
= & 0.0000 \\
= & 0.5871 \\
= & .39912\end{array}$ \\
\hline 1nsalario & coef. & $\begin{array}{l}\text { Robust } \\
\text { std. Err. }\end{array}$ & $t$ & $P>|t|$ & [95\% conf. & Interva1] \\
\hline $\begin{array}{r}\text { exper } \\
\text { civi } 1 \\
\text { genero } \\
\text { estrat } \\
\text { Multinac } \\
\text { Gerente } \\
\text { Director } \\
\text { _cons }\end{array}$ & $\begin{array}{r}.0399092 \\
-.1635617 \\
.1816492 \\
.2057326 \\
.2875214 \\
.4875685 \\
.5341092 \\
14.31679\end{array}$ & $\begin{array}{r}.0073368 \\
.08274 \\
.0758662 \\
.0881391 \\
.0686062 \\
.1195858 \\
.0956432 \\
.1331595\end{array}$ & $\begin{array}{r}5.44 \\
-1.98 \\
2.39 \\
2.33 \\
4.19 \\
4.08 \\
5.58 \\
107.52\end{array}$ & $\begin{array}{l}0.000 \\
0.050 \\
0.018 \\
0.021 \\
0.000 \\
0.000 \\
0.000 \\
0.000\end{array}$ & $\begin{array}{r}.0253962 \\
-.3272296 \\
.0315785 \\
.0313847 \\
.1518114 \\
.2510159 \\
.3449175 \\
14.05339\end{array}$ & $\begin{array}{r}.0544222 \\
.0001061 \\
.33172 \\
.3800805 \\
.4232313 \\
.7241211 \\
.7233008 \\
14.58019\end{array}$ \\
\hline
\end{tabular}

Fuente: Autores. Salida STATA.

En cuanto a la sensibilidad de los coeficientes de las variables estrato, género y estado civil, se observa un cambio en la importancia de las variables de género y estado civil con respecto a la regresión anterior, pues en la regresión definitiva gana importancia para explicar los salarios el género mientras que el estado civil la pierde. En lo que tiene que ver con la variable de estrato, ésta sigue conservando su importancia.

\section{Conclusiones}

Los resultados obtenidos por el modelo de regresión muestran que los principales factores que inciden en una mejor remuneración salarial de los contadores públicos egresados de la Universidad EAFIT son en orden de importancia: el cargo laboral desempeñado (quienes se ejercen como directores y gerentes perciben primas en sus remuneraciones salariales de $70,59 \%$ y $62,84 \%$ ), el estar empleado en empresas multinacionales (lo cual representa una prima de 33,31\%), el vivir en estratos altos (lo cual mejora la remuneración en un $22,84 \%$ ), el pertenecer al género masculino (quienes perciben un $19,92 \%$ más de salario que las mujeres), el haberse involucrado en el pasado o en el presente, en una relación sentimental (este grupo de individuos percibe una prima en sus salarios de $17,77 \%$ ), y por último, el poseer un mayor número de años de experiencia profesional (cada año adicional de experiencia profesional proporciona una retribución en los salarios de 4,07\%).

Se puede afirmar que por lo menos en el caso de un grupo de individuos con características de formación académica muy similares (quienes poseen un pregrado universitario), la teoría del capital humano, específicamente la lógica presentada por la ecuación de Mincer (1976) parece no aplicar. Es decir que, las variables más relacionadas con la medición de la productividad laboral de un individuo, el número 
de años de educación formal y la experiencia laboral no son tan influyentes en el comportamiento de los salarios de los sujetos bajo estudio como lo predice la teoría. Por el contrario, en la explicación de la remuneración laboral de los contadores eafitenses presenta una mayor importancia variables relacionadas con la demanda laboral como son los cargos, y el ámbito de la empresa, así como variables de tipo socioeconómico de los individuos como son el estrato socioeconómico, muchas veces relacionado con la clase social, el género y el estado civil.

El análisis anterior conlleva entonces a plantearse la siguiente pregunta: ¿Realmente los cambios en los niveles de educación en una sociedad conllev an a cambios en la inequidad en el ingreso? Probablemente existe una relación causal entre estas dos variables, y por lo tanto, podría decirse que en una sociedad en la cual la inversión en educación es alta, pueden esperarse niveles de equidad mayores. Sin embargo, trabajos empíricos como este muestran que es necesario avanzar aún más en la investigación acerca del tema.

\section{Referencias}

Card, D. (1999). The causal effect of education earnings. Berkeley, California: Elsevier Science B.V.

Cardona, M., Montes, I., Vásquez, J. J., y Villegas, M. Y. (2007). Capital Humano: una mirada desde la educación y la experiencia laboral. Cuadernos de Investigación EAFIT Semillero de investigación en economía, Documento No 56, 1-40.

Heckman, J. J. (1979). Sample selection bias as a specification error. Econometrica, 1 (47), 153 - 161.

Hidalgo, A. (1999). Educación y rentas, una aplicación al mercado de los economistas. España: Universidad de Castilla - La mancha.

Lassibille, G., y Navarro Gómez, M. L. (2004). Manual de economía de la educación. Madrid: Ediciones Pirámide.

Mincer, J. (1974). Schooling, Experience and Earnings. Columbia: Columbia University Press.

Prada, C. F., y Vélez, C. E. (2006). ¿Es rentable la decisión de estudiar en Colombia? Ensayos sobre política económica, No. 51 Banco de la República Bogotá, 226 329.

Salas, M. (2001). Aspectos económicos de la educación. En M. Salas (Ed.), Aspectos económicos de la educación (pp. 185-219). España: Grupo Editorial Universitario. 
Salas, M. (2003). Determinantes salariales. En: el Mercado laboral de los titulados Universitarios. X Jornadas de la Asociación de Economía de la Educación. Granada, España.

Tenjo, J. G., Ribero, R. M., y Bernat, L. F. (2005). Evolución de las diferencias salariales por sexo en seis países de América Latina un intento de interpretación. Documento CEDE. 